\title{
Medical records confidentiality and public health research: two values at stake? An italian survey focus on individual preferences
}

\author{
Virgilia Toccaceli, Corrado Fagnani, Maria Antonietta Stazi \\ Genetic Epidemiology Unit, National Centre of Epidemiology, Surveillance and Health \\ Promotion, Italian National Institute of Health, Rome, Italy
}

\begin{abstract}
Significance for public health
Information retrieved from medical records is critical for public health research and policy. In particular, large amounts of individual health data are needed in an epidemiological setting, where methodological constraints (e.g. follow-up update) and quality control procedures very often require data to be re-identifiable. Concern about European regulation affecting access to medical records seems to be widespread in the scientific community. Highlighting individuals' concerns and preferences about privacy and informed consent regarding the use of health data can support policy making for public health research. It can contribute to the design of procedures aiming to extract the greatest value from medical records and, more importantly, to create a system for the protection of personal data tailored to the needs of different people.
\end{abstract}

\begin{abstract}
In a time when Europe is preparing to introduce new regulations on privacy protection, we conducted a survey among 1700 twins enrolled in the Italian Twin Register about the access and use of their medical records for public health research without explicit informed consent. A great majority of respondents would refuse or are doubtful about the access and use of hospital discharge records or clinical data without their explicit consent. Young and female individuals represent the modal profile of these careful people. As information retrieved from medical records is crucial for progressing knowledge, it is important to promote a better understanding of the value of public health research activities among the general population. Furthermore, public opinions are relevant to policy making, and concerns and preferences about privacy and confidentiality in research can contribute to the design of procedures to exploit medical records effectively and customize the protection of individuals' medical data.
\end{abstract}

\section{Introduction}

Two distinct trends are easily detectable in Europe regarding privacy and public health research. On the one hand, we have a European data protection regulation that, after an amendment voted in October 2013, will introduce restrictive rules concerning the use of personal data for scientific research; ${ }^{1,2}$ on the other hand, Europe is challenging a huge effort to develop as quickly as possible large networks of individual medical information (often linked to biological materials) for epidemiological and biomedical research (e.g: BBMRI, Biobanking and Biomolecular Resources Infrastructure; EPIC Project, The European
Prospective Investigation into Cancer and Nutrition). The use of health data, in particular information retrieved from medical records, is crucial to public health research and policy. Follow-up, record-linkage and quality control procedures require data to be re-identifiable, and it may not be always feasible to implement informed consent procedures every time data is used for research purposes. Concerns about the European Union's forthcoming regulation of access to medical records seem to be widespread in the international scientific community. ${ }^{1-5}$ It is noticeable that a general balance between privacy protection and public health interests remains an urgent priority both at national level, where the protection of personal data is strictly regulated by the legislative decree n. 196, 30 June 2003, and at international level. In this context, we considered it of value to investigate the preferences of lay people. As our group works at the Italian Twin Register (ITR), ${ }^{6}$ we conducted an opinion survey among the twins enrolled in this population-based Register. The ITR, like other Twin Registers worldwide, is a valuable tool for epidemiological studies essential to the development of health prevention plans (http://www.iss.it/gemelli/index.php? lang=1). The survey investigated the opinion of adult twins resident in Italy about the potential access to and use of their medical records by researchers without specific informed consent.

\section{Design and methods}

\section{Ethics and approval}

This survey forms part of a series of studies on the ELSI (ethical, legal, and social issues) of the epidemiological research and biobanking activities conducted by the ITR. Ethical approval was given by the Ethics Board of the Italian National Institute of Health, on the $21^{\text {st }}$ of January 2003.

\section{Study population}

The survey was conducted with a self-administered questionnaire. Participants are adult twins who enrolled in the ITR over a 12-year period (2001-2012). A total of nearly 5000 twins aged 18-70 years, resident all over Italy were mailed, between June and October 2012, the questionnaire along with an informed consent form and a letter explaining the objectives of the survey.

\section{Questionnaire}

The outcome in this report is the theoretical agreement of respondents on the access and use of their medical records (e.g., patients health data recorded by general practitioners, hospital discharge records) for research purposes without informed consent (English translation of the Italian questionnaire item: Do you agree that your 
medical data, available from previous check-ups and/or hospital admissions, can be accessed and used for research purposes without your explicit informed consent?). Socio-demographic data (i.e. age, gender, education, marital status, geographical area of residence) were recorded as well as self-perceived health, self-reported presence of chronic or long term diseases, and present or past job experience in the healthcare sector. These items were part of a larger survey on attitude towards research and willingness to donate biological material for research biobanks. ${ }^{7}$ Based on an overview of the international literature on the ethical, legal and social issues of biomedical research, we developed a preliminary version of the questionnaire, that we submitted to a group of experts (three psychiatrists, one bioethicist) at the Italian National Institute of Health to assess content validity; all of them agreed that the questionnaire was a useful measure of opinions and concerns on research and biobanking and that most of the questions were both relevant and important. We then performed a testretest reliability estimation (based on Cohen's kappa statistic) on a convenience sample of 34 subjects who were administered the questionnaire twice, at a distance of 14 days. The degree of agreement between the two assessments was found to be adequate for most of the items; a few items showed poor agreement and were thus modified before inclusion in the final version of the questionnaire. A more detailed description of the design and validation processes of this selfreport instrument can be found elsewhere. ${ }^{7}$

\section{Statistical methods}

We first derived univariate sample statistics (means/ranges and percentages) considering twins as individual units. Then, we used ANOVA and chi-square tests to identify socio-demographic factors potentially associated with the study outcome. Finally, to address possible confounding effects in crude associations, we fitted a multinomial logistic regression model, using robust estimation of standard errors (as implemented in the Stata software 13.0) to take account of non-independence of data within twin pairs.

\section{Results}

Respondents were 1693 (response rate 35\%). There were no significant differences in age, gender, education and marital status between respondents and non-respondents. The study population included about $60 \%$ of women. The median age was 38 years (range: 18-70 years). More than one-third of individuals had an education above high school. A percentage of $56 \%$ of respondents were against the access and use of their medical records without their specific informed consent, $33 \%$ were in favour, while $11 \%$ had no clear opinion about it. More than $10 \%$ declared a present or past work experience in the healthcare sector. Furthermore, health status was perceived good or very good by more than $85 \%$ of the subjects, and $80 \%$ reported no chronic or long term diseases (Tables 1 and 2).

Age, gender, self-reported presence of chronic or long term diseases, and present or past job in the healthcare sector were significantly associated (ANOVA and chi-square tests) with the study outcome (data not reported). Therefore, we included these variables as explanatory factors in the multinomial logistic regression model, along with education, residence, marital status and self-perceived health as possible confounding factors. Age, gender and work experience in the healthcare sector remained significant after simultaneous model-based adjustment (Table 3). In particular, the frequency of theoretical agreement on the access and use of personal medical records without an explicit informed consent increased with the increase of age; the agreement probability was $40 \%$ lower for women and for individuals who declared a present or past employment in the healthcare field.
Table 1. Demographics of the study population.

\begin{tabular}{lc} 
Demographic variables & N. (\%) \\
Age in years* & $38(18-70)$ \\
Gender & \\
Male & $664(39.2)$ \\
Female & $1029(60.8)$ \\
Education & \\
Primary school & $33(1.9)$ \\
Secondary school & $200(11.9)$ \\
Vocational school & $142(8.4)$ \\
High school & $673(39.9)$ \\
3-year degree & $153(9.1)$ \\
5-year degree & $485(28.8)$ \\
\hline Marital status & \\
Single & $901(53.4)$ \\
Married & $585(34.7)$ \\
Living in couple & $103(6.1)$ \\
Separated & $41(2.4)$ \\
Divorced & $45(2.7)$ \\
Widowed & $12(0.7)$ \\
Area of residence & \\
North & $918(54.7)$ \\
Centre & $566(33.7)$ \\
South & $195(11.6)$ \\
\hline *Median (range). &
\end{tabular}

Table 2. Questionnaire results.

Questionnaire items N. (\%)

Agreement on access and use of medical records

for research without explicit informed consent

\begin{tabular}{lc} 
No & $949(56.5)$ \\
Yes & $551(32.8)$ \\
I don't know & $180(10.7)$ \\
esent or past work experience in the health care sector & \\
No & $1491(88.6)$ \\
Yes & $192(11.4)$ \\
\hline elf-perceived health & \\
Very bad & $3(0.2)$ \\
Bad & $19(1.1)$ \\
Fair & $200(11.9)$ \\
Good & $1045(61.9)$ \\
Very good & $421(24.9)$ \\
Neff-reported presence of chronic or long term diseases & \\
No & $1350(80.0)$ \\
Yes & $337(20.0)$ \\
\hline
\end{tabular}

Number of subjects (N) for each variable may not sum up to total (1693) due to missing information.

Table 3. Multinomial logistic regression analysis of the theoretical agreement of respondents on the access and use of their medical records for epidemiological and biomedical research purposes without an informed consent.

\begin{tabular}{lccc} 
Subjects agreement & OR & P-value & $95 \% \mathrm{CI}^{*}$ \\
Age (years) & 1.024 & $<0.001$ & $(1.012,1.036)$ \\
$\begin{array}{l}\text { Gender } \\
\quad \text { Male }\end{array}$ & 1 & & \\
$\quad$ Female & 0.605 & $<0.001$ & $(0.480,0.763)$ \\
\hline
\end{tabular}

Present or past work experience in the health care sector

$\begin{array}{lccc}\text { No } & 1 & & \\ \text { Yes } & 0.618 & 0.010 & (0.427,0.892)\end{array}$

OR: odds ratio; $95 \% \mathrm{CI}$ : 95\% confidence interval. * Standard errors were adjusted for clustering within twin pairs. Outcome coding: $1=Y e s$, I agree, $0=$ No, I do not agree, $8=I$ don't know. Results of I do not know vs No, I do not agree are not reported. The model was also adjusted for education, area of residence, marital status, self-perceived health and self-reported presence of chronic or long term diseases. 


\section{Discussion and conclusions}

We consider this survey an important source of information on this worldwide, high priority topic, and, to our knowledge, it is at present the only one conducted in Italy. It is worth pointing out that twins have been shown to be representative of non-twin individuals for several social and behavioural traits (e.g. political attitudes and behaviours), ${ }^{8}$ and so the results of this survey are likely to provide a reliable picture of general population concerns with respect to public health research and privacy.

The proportion of individuals who would object to the access and use of their medical records without explicit informed consent is fairly high. We could reasonably assume that concerns regarding confidentiality and privacy protection are the main reasons behind this prevailing opinion. Following this line of reasoning, we would have expected that, among individuals who are already enrolled in an epidemiologyoriented registry, and thus generally willing to participate in such activities, these concerns would be less widespread. In this perspective, our results could even be conservative estimates of the level of concern about privacy and confidentiality among the general population.

In particular, younger and female individuals seem to represent the modal profile of respondents who are not favourable to the access of their medical data or doubtful about this when a specific informed consent is not proposed. It is noteworthy that a similar gender effect was also detected in a previous study, ${ }^{9}$ where women showed higher level of awareness and alertness regarding biological samples donation for research purposes. Of note is also the fact that those individuals with healthcare sector work experience showed a significantly lower level of acceptance to access of medical records without explicit informed consent; a variety of interpretations could be proposed, and they would deserve further specific investigations. However, considering the cultural background in Italy, it is our opinion that the attitude of health care personnel in such issues is important to promote trust in public health research among the general public. On the whole, as this reluctance seems to be widespread and the use of health data is essential for public health research, it would be highly advisable, from a social and ethical perspective, to promote similar surveys among the general population at national and international level.

We are aware of a few limitations of our research. The response rate, though among the highest rates obtained in the ITR surveys, is far from optimal. Nevertheless, the socio-demographic homogeneity between respondents and non-respondents should preclude the major sources of selection bias. The fact that health status was perceived good or very good by more than $85 \%$ of the subjects, and that $80 \%$ reported no chronic or long term diseases draws attention to the possible self-selection of healthy individuals to the survey. However, our multivariate model was adjusted by both self-perceived and self-reported health status, therefore the effects of such a bias on our findings are likely to be modest. Last but not the least, the survey instrument itself can only give a preliminary view when public opinions are investigated. To explore more thoroughly privacy concerns in public health research, the approach should be qualitative. When the goal concerns policies involving ethical dimensions, more appropriate methodologies are interviews, focus groups and deliberative democracy. Nevertheless, the results of this survey can pave the way to future research which will capture motivations behind responses and differences depending on contextual factors more efficiently. Our results seem to confirm what is being considered as the privacy paradox, ${ }^{10}$ that is a certain conflict between individuals' motivations for consenting to specific use of personal data and their fear about unknown disclosure of these data, especially in the framework of contemporary major IT developments. This paradox has been currently detected in Europe; also in the USA, the Institute of Medicine has recently reported that only about one out of
10 Americans supports sharing medical data with researchers without consent. ${ }^{11}$

Public opinions are relevant to policy making; in our case, concerns and preferences of individuals can contribute to the design of procedures aiming to effectively exploit medical records for public health research and, more importantly, to tailor the protection of individuals' medical data. What is more, there is a clear need, well detected in Europe ${ }^{5}$ to promote among people a better understanding of the importance of public health research, that is performed by the use of health data, and in particular of large numbers of medical records. Assuming - as we did - that privacy concerns basically drove the response to our survey outcome, we agree with Johnsson and colleagues ${ }^{12}$ that, beyond the legal efforts to protect individual privacy, it is central to address the responsibility and moral commitment of researchers and health care personnel. When public health activities are concerned, no new legal framework will be effective if moral education of researchers and operators is not pursued.

In conclusion, this survey highlights the concerns of more than $56 \%$ of 1693 Italian respondents who would object to the use of their medical records in research without specific informed consent; an efficient personalization of medical data protection emerges as an urgent policy to be developed for public health.

Correspondence: Virgilia Toccaceli, Genetic Epidemiology Unit, National Centre of Epidemiology, Surveillance and Health Promotion, Italian National Institute of Health, viale Regina Elena 299, 00161 Rome, Italy.

Tel.: +39.06 .4990 .4178 - Fax: +39.06 .4990 .4151 .

E-mail: virgilia.toccaceli@iss.it

Key words: privacy and confidentiality, medical records, public health, twins, use of personal data in research.

Acknowledgments: the authors are grateful to all the twins who took part in the study. We acknowledge Cristina D'Ippolito, Antonio Arnofi, Sabrina Alviti and Miriam Salemi for their skilful assistance in the database management of the Italian Twin Register.

Contributions: VT, MAS, study concept and design; CF, statistical analysis; VT, CF, drafting of the manuscript; VT, CF, MAS, critical revision of the manuscript for important intellectual content; VT, MAS, study supervision; VT, CF MAS, final approval.

Conflict of interests: the authors declare no potential conflict of interests.

Received for publication: 13 October 2014.

Accepted for publication: 18 February 2015.

(C) Copyright V. Toccaceli et al., 2015

Licensee PAGEPress, Italy

Journal of Public Health Research 2015;4:401

doi:10.4081/jphr.2015.401

This work is licensed under a Creative Commons Attribution NonCommercial 3.0 License (CC BY-NC 3.0).

\section{References}

1. Dolgin E. New data protection rules could harm research, science groups say. Nat Med 2014;20:224.

2. Seniori Costantini A, Saracci R. EU: più privacy e meno ricerca? Epidemiol Prev 2014;38:4.

3. Wartengberg D, Thompson WD. privacy versus public health: the impact of current confidentiality rules. Am J Public Health 2010;100:407-12.

4. Allen J, Homan CD, Meslin EM, et al. Privacy protectionism and health information: is there any redress for harms to health? J Law Med 2013;21:473-85. 
5. Olsen J. Data Protection and epidemiological research: a new EU regulation is in the pipeline. Int J Epidem 2014;1353-54.

6. Brescianini S, Fagnani C, Toccaceli V, et al. An update on the Italian Twin Register: advances in cohort recruitment, project building and network development. Twin Res Hum Gen 2013;16:190-6.

7. Toccaceli V, Fagnani C, Gigantesco A, et al. Attitudes and willingness to donate biological samples for research among potential donors in the Italian Twin Register. J Empir Res Hum Res Ethics 2014;9:39-47.

8. Klemmensen R, Hobolt SB, Dinesen PT, et al. The Danish political twin study: political traits in Danish twins and the general population. Twin Res Hum Gen 2012;15:74-8.

9. Toccaceli V, Fagnani C, Nisticò L, et al. Research understanding, attitude and awareness towards biobanking: a survey among Italian twin participants to a genetic epidemiological study. BMC Med Ethics 2009;10:4.

10. Büschel I, Mehdi R, Cammilleri A, Marzouki Y, Elger B. Protecting human health and security in digital europe: how to deal with the privacy paradox? Sci Eng Ethics 2014;20:639-58.

11. Caine K, Hanania R. Patients want granular privacy control over health information in electronic medical records. J Am Med Inform Assoc 2013; 7-15.

12. Johnsson L, Eriksson S, Helgesson G, et al. Making researchers moral: Why trustworthiness requires more than ethics guidelines and review. Res Ethics 2014;10:29-46. 